PALEO

Revue d'archéologie préhistorique

28 | 2017

Varia

\title{
Jacques Brunet (1942-2017)
}

Philippe Malaurent et Dominique Baffier

\section{(2) OpenEdition}

Journals

Édition électronique

URL : http://journals.openedition.org/paleo/3065

DOI : $10.4000 /$ paleo.3065

ISSN : 2101-0420

Éditeur

SAMRA

Édition imprimée

Date de publication : 30 décembre 2017

Pagination : 9-13

ISSN : 1145-3370

Référence électronique

Philippe Malaurent et Dominique Baffier, « Jacques Brunet (1942-2017)», PALEO [En ligne], 28 | 2017 mis en ligne le, consulté le 15 septembre 2020. URL : http://journals.openedition.org/paleo/3065

\section{(C) $\oplus \Theta \Theta$}

PALEO est mis à disposition selon les termes de la licence Creative Commons Attribution - Pas d'Utilisation Commerciale - Pas de Modification 4.0 International. 


\section{Jacques BRUNET (1942-2017)}

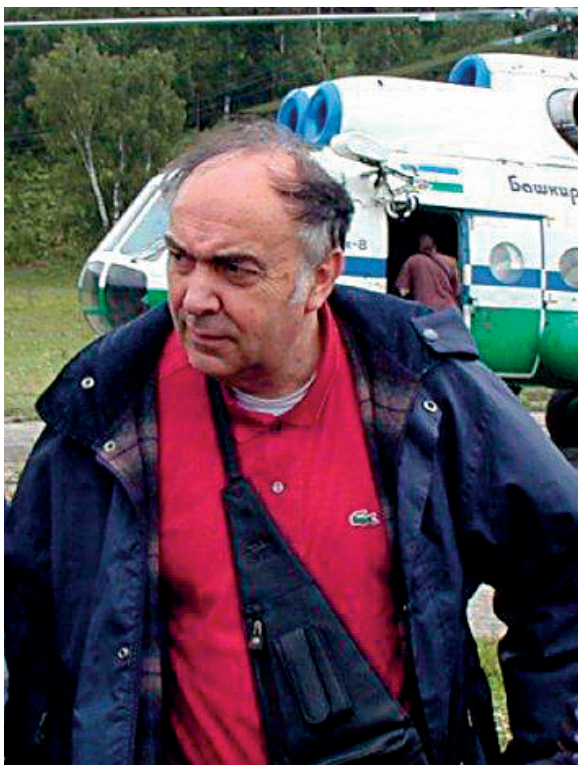

Une fois de plus le grand Taureau de Lascaux pleure. Jacques Brunet nous a quitté le 29 mai dernier. Jacques s'engagea très tôt dans la conservation préventive du patrimoine souterrain orné ; responsable du pôle « Grottes ornées 》 de 1974 à 2006 au LRMH, il parcourut les sites de l'Afrique du Nord, de l'Oural, de l'Amérique centrale et de l'Europe pour dispenser conseils et savoirs.

Jacques a beaucoup œuvré pour la conservation de Font de Gaume, Combarelles, Rouffignac, Lascaux et tout autant pour un grand nombre d'autres grottes françaises ou étrangères qu'il serait interminable de citer ici. II s'est également consacré à la conservation d'abris sous roche comme, par exemple, celui de Cap Blanc et à des sites en plein air comme Foz Côa au Portugal. Je ne rentrerai pas dans une guerre picrocholine, je demanderai seulement à ses protagonistes de voir, revoir, analyser ses travaux qui ont donné lieu à de nombreux rapports, publications, livres et je ne citerai que deux ouvrages faisant toujours références : «Conservation Des Grottes Ornées ; Éditeur : CNRS » et «Conservation de l'Art rupestre ; Études et Documents sur le Patrimoine culturel ; UNESCO ».

Jacques laissera l'image d'un homme modeste, réservé et cultivé, qu'il faisait bon côtoyer.

Philippe MALAURENT

\section{Nous l'appelions Jacqueusse !}

L'annonce de la disparition de Jacques Brunet m' a surprise et profondément peinée. Il est parti sans bruit, discrètement, sans importuner quiconque, un peu comme il semblait être dans la vie. Nous avions pris l'habitude de nous téléphoner assez régulièrement pour échanger quelques nouvelles de «la boutique», comme il disait, et nous réconforter sur notre état de santé mutuel. Mais ces derniers mois, le téléphone sonnait dans le vide. Je l'ai imaginé à la montagne chez son frère, mais l'absence avait une durée inhabituelle et puis... le téléphone n'a plus sonné !

Ingénieur de recherche au Laboratoire de recherche des Monuments historiques, il était responsable du pôle des grottes ornées et mettait tout en œuvre pour assurer la pérennité de ces espaces patrimoniaux fragiles. II se retrouve associé aux recherches faites dans les grottes ornées françaises les plus prestigieuses : Lascaux, Font-de-Gaume, Rouffignac, Niaux, Chauvet, Arcy-sur-Cure, etc. Dans les nombreuses publications, son nom est le plus souvent associé à ceux de P. Vidal, J. Vouvé et parfois Ph. Malaurent. Ce groupe des quatre, les mousquetaires des grottes ornées, poursuivait en effet des recherches convergentes qui avaient pour but la sauvegarde et la conservation de l'art rupestre ainsi que la compréhension des phénomènes susceptibles d'être porteurs de dégradation. 
Souvent aussi Jacques Brunet partait à l'étranger participer à des colloques, donner des cours dans des Universités, des conférences. II aimait le dépaysement, II était souvent consulté par l'UNESCO, en tant qu'expert, pour apporter son expérience et ses conseils pour la sauvegarde de sites étrangers en péril. De ces voyages, ce fut celui qu'il effectua au Japon qui semblait l'avoir marqué le plus profondément. II en parlait de lui-même, avec enthousiasme, en oubliant un peu de sa réserve habituelle.

Pendant plus de 20 ans, nous avons eu un rendez vous annuel à la Grande Grotte d'Arcy-sur-Cure. II aimait venir suivre nos travaux. Depuis le début de notre étude il nous avait accompagnés et conseillés dans des choix souvent difficiles. Sa présence était discrète, il marchait à petits pas dans la salle des Vagues en toussotant et en attendant avec patience que nous lui montrions les dernières découvertes. II ne s'imposait pas, il écoutait, puis proposait et convainquait. II était respectueux des autres. II aimait la grotte, il l'auscultait et avec sa conviction tranquille, il avait même réussi à persuader le propriétaire de supprimer les éclairages trop violents et de remplacer l'eau de javel à forte concentration par un traitement plus doux et plus approprié à la conservation des peintures.

C'était un chercheur attentif au milieu souterrain mais qui n'était pas atteint de frilosité. II était prêt à s'engager dans des projets nouveaux, et se lancer avec ardeur mais raison dans l'aventure après avoir effectué toutes les vérifications et les essais qui prouvaient l'innocuité de la démarche. Il était d'une efficacité sans faille et trouvait toujours les bonnes personnes et les bonnes compétences au bon endroit. Ainsi pour l'amincissement de la calcite, il avait, avec nous, monté le dossier d'intervention sur les parois ornées qui devait être présenté aux plus hautes instances du Ministère de la Culture. II était venu faire des essais avec Eudald Guillamet, restaurateur andorran, qui avait déjà travaillé dans une grotte ornée puisqu'il était intervenu sur le plafond de Rouffignac, en Dordogne, pour en éliminer les graffitis. Le dossier d'Arcy a été accepté et l'aventure a commencé, aventure longue et méticuleuse qui a permis de révéler plus de 200 unités graphiques datées de $28000 \mathrm{BP}$, dans un état de conservation étonnant et toujours préservées par deux couches distinctes de calcite.

C'est à Eudald qu'il devait son surnom, car en Andorre on prononce toutes les lettres quand on parle ! et sans accent s'il vous plaît ! Ce qui, toujours sans accent, donne Jacqueusse Brrrounette ! II riait avec nous de cette plaisanterie répétitive et sélective, car il était le seul à être affublé de cette appellation andorrane protégée. II riait, rarement aux éclats, mais toujours un peu dans sa «barbe», la tête légèrement penchée en avant avec un regard de côté, piquant et débordant d'humour. Parfois, les premiers mots se bousculaient, le discours se faisait haché et ponctué de petits toussotements. Tes doigts, toujours en mouvement, tapotaient la table ou rassemblaient les miettes de pain et tu étais là, avec nous, entouré de rires et on te voyait, heureux d'être là, associé en ami à nos grandes tablées.

Grâce à toi, Jacques, grâce à ton engagement, grâce à ton professionnalisme, nous avons pu réaliser ensemble un travail innovant, " une première mondiale », comme disait le Comte de la Varende, propriétaire des lieux, qui a permis de ressusciter ces peintures, mais aussi de développer d'autres recherches, en particulier sur la calcite, son identification, sa croissance et les possibilités d'intervenir sur celle-ci. Encore une nouvelle démarche pour la protection des grottes ornées dont tu es indirectement à l'origine.

Cette année, nous t'avons attendu en vain sur le parking de la grotte, pour t'« aider », comme d'habitude, à sortir tes bottes de ton coffre. Te souviens-tu de ces bottes étranges, dont chacune d' elle contenait toujours une bouteille de vieux bordeaux ??

Au revoir Jacqueusse, notre amitié est en deuil.

Dominique BAFFIER

\section{Bibliographie}

AUJOULAT Norbert, BRUNET Jacques et VIDAL Pierre et al. 1990 - Chroniques des années de pierre... : art préhistorique-sciences et techniques modernes - Meyzieu : Césura Lyon. - 1 vol. (127 p.).

AUJOULAT Norbert, ANDRIEUX Claude, MANGIN Alain, CLOTTES Jean, SUMERLY Frédéric, MENU Michel, WALTER Philippe, LORBLANCHET Michel, BRUNET Jacques, VIDAL Pierre, CHEVILLOT Christian, ROUZAUD François et SIGAUD Monique 1990 - Archéologie des grottes ornées : Montignac 1990 - Paris : Ministère de la Culture, de la Communication, des Grands Travaux et du Bicentenaire, Direction du Patrimoine, Sous-Directions de
l'Archéologie et des Monuments Historiques ; Périgueux : Conseil Général de la Dordogne. 1 vol. (47 p.).

BAFFIER Dominique, GIRARD Michel, BRUNET Jacques et al. 2001 - Étude et conservation de l'art pariétal : exemple de la Grande Grotte d'Arcy-sur-Cure (Yonne) et al. Techné, 13-14, p. 63-70.

BAFFIER Dominique, GIRARD Michel, BRUNET Jacques et al. 2001 - Découvertes : du nouveau à la Grande Grotte d'Arcy-sur-Cure, Yonne, France - International newsletter on rock art, 28, p. 1-3.

BROMBLET P., BRUNET J. 1997 - L'Étude de faisabilité, condition nécessaire à la réalisation de l'estampage de parois sculptées : l'exemple de l'Abri Bourdois du Roc-aux- 
Sorciers (Angles-sur-l'Anglin, Vienne, France) International newsletter on Rock art, 18, p. 21-25.

BRUNET Jacques, BROMBLET Philippe 1998 - Le Rocaux-Sorciers, Angles-sur-l'Anglin, Vienne. Étude de faisabilité du moulage de sculptures préhistoriques Monumental, 20, p. 80-84.

BRUNET Jacques, CLOTTES Jean, GENESTE JeanMichel et al. 2006 - Les Grottes ornées. Monumental, 20062, p. 5-104.

BRUNET Jacques, DEMAILLY Sylvie, VIDAL Pierre 1989 Résultats de l'étude de prélèvements de peintures des abris du Tassili N'Ajjer - Ars Praehistorica, 1988-1989, vol. 7-8, p. 293-303.

BRUNET Jacques, GUILLAMET Eudald, PLASSARD Jean 1997 - L'Élimination des graffitis de Rouffignac. International newsletter on Rock art, 17, p. 11-15.

BRUNET Jacques, GUILLAMET Eudald, PLASSARD Jean et VIDAL Pierre 1996 - Réhabilitation des œuvres préhistoriques de Rouffignac - Paris : Errance ; Section Française de l'Institut International de Conservation. (CoRé, Conservation et Restauration du Patrimoine Culturel ; $\left.n^{\circ} 1\right)$. - p. 5-9.

BRUNET Jacques, MALAURENT Philippe et VOUVÉ Jean 1997 - Lascaux : histoire d'un difficile sauvetage Archéologia, 332, p. 24-35.

BRUNET Jacques, MALAURENT Philippe, VOUVÉ Jean 1998 - De Lascaux à la grotte Chauvet : comment conserver l'art pariétal - L'Archéologue, Archéologie Nouvelle, 33, p. 41-45.

BRUNET Jacques, MARSAL Jacques, VIDAL Pierre 1980 Lascaux ; où en sont les travaux de conservation ? Archéologia, 149, p. 35-50.

BRUNET Jacques, VIDAL Pierre 1981 - Conditions biologiques - Monuments Historiques, 118, p. 54-62.

BRUNET Jacques, VIDAL Pierre 1981 - Font de Gaume aux Eyzies, les derniers travaux de conservation. Archéologia, 161, p. 19-32.

BRUNET Jacques, VIDAL Pierre 1981 - Grotte du PechMerle, Cabrerets (Lot) : étude des conditions climatiques à proximité de la Frise Noire. Conséquences pour la conservation. In : Congrés préhistorique de France : compte rendu de la XXl ${ }^{\text {e }}$ session, Quercy, 3-9 septembre 1979. Paris, tome 1. p. 71-84.

BRUNET Jacques, VIDAL Pierre 1984 - Surveillance et mesures de protection - In : L'Art des cavernes : Atlas des grottes ornées paléolithiques françaises. Paris, Imprimerie Nationale, p. 57-62.

BRUNET Jacques, VIDAL Pierre 1989 - Conservation des grottes ornées - In : Art pariétal paléolithique : Études et conservation. Actes du colloque international d'art pariétal paléolithique tenu à Périgueux-Le Thot du 19 au 22 novembre 1984. Périgueux - Centre National de Préhistoire ; Paris, Ministère de la Culture et de la Communication, des grands Travaux et du Bicentenaire. Actes des colloques de la Direction du patrimoine, 6, p. 179-191.

BRUNET Jacques, VIDAL Pierre 1989 - Le problème du seuil de fréquentation - In : Art pariétal paléolithique : Études et conservation. Actes du colloque international d'art pariétal paléolithique tenu à Périgueux-Le Thot du 19 au 22 novembre 1984. Périgueux - Centre National de Préhistoire ; Paris, Ministère de la Culture et de la Communication, des grands Travaux et du Bicentenaire. Actes des colloques de la Direction du patrimoine, 6, p. 229-239.

BRUNET Jacques, VIDAL Pierre 1989 - Jacques Marsal, inventeur de Lascaux- Archeologia, 250, p. 4-5.

BRUNET Jacques, VIDAL Pierre, STEFANAGGI Marcel 1980 - Grotte de Font de Gaume aux Eyzies (Dordogne) : étude du taux de gaz carbonique de l'atmosphère. Conséquences pour la conservation des figurations préhistoriques - [Oviedo] : [Universidad de Oviedo, Facultad de Ciencas, Instituto de Geología Aplicada] ; [Barcelona] : [Centre Excursionista de Catalunya, Club Alpí Catalá]. - (Speleon ; 25). - p. 37-45.

BRUNET J., VIDAL P., VOUVÉ J. 1985 - Conservation de l'art rupestre : deux études, glossaire illustré. - [S.I.] : Unesco. - 1 vol. (108 p.) - (Études et documents sur le patrimoine culturel ; 7).

BRUNET Jacques, VIDAL Pierre, VOUVÉ Jean 1986 Réflexions sur la conservation du patrimoine souterrain. Documents d'Archéologie Périgourdine, 1, p. 7-16.

BRUNET Jacques, VIDAL Pierre et VOUVÉ Jean 1987 The Conservation of rock art - Paris : UNESCO. - (Studies and documents on the cultural heritage ; 7).

BRUNET Jacques, VIDAL Pierre et VOUVÉ Jean 1990 Nouveau bilan sur la conservation des œuvres pariétales de Lascaux. In : L'archéologie des grottes ornées. Cinquantenaire de la découverte de la grotte de Lascaux : résumés des communications du colloque international tenu à Montignac du 10 au 14 septembre 1990 - SousDirection de l'archéologie et des monuments historiques : Conseil général de la Dordogne. - p. [19].

BRUNET Jacques, VIDAL Pierre, VOUVÉ Jean 1990 - La conservation de Lascaux - Dossiers d'Archéologie, 152, p. 68-75.

BRUNET Jacques, VIDAL Pierre, VOUVÉ Jean 1990 - La conservation de l'art rupestre dans le monde - Archeologia, 261, p. 4-6.

BRUNET Jacques, VOUVÉ Jean (dir.) 1996 - La Conservation des grottes ornées. - Paris : CNRS. - 1 vol. (263 p.). - (Conservation du patrimoine ; 4). 
BRUNET Jacques, VOUVÉ Jean, MALAURENT Philippe 1999 - La grotte Cosquer : une problèmatique insolite. L'accès artificiel à la grotte préhistorique $\mathrm{H}$. Cosquer est-il compatible avec le maintien et le respect des conditions de conservation ? - CoRé : Conservation et restauration du patrimoine culturel, 7 (novembre 1999), p. 39-45.

BRUNET Jacques, VANRELL Luc, MALAURENT Philippe, VOUVÉ Jean 2002 - La grotte Cosquer : une cavité sous pression - In : L'art avant l'histoire, la conservation de l'art préhistorique / $10^{\text {mès }}$ journées d'études de la section française de l'institut international de conservation. Paris, 23-24 mai 2002. - Champs-sur-Marne : SFIIC. p. 43-48.

DAUBISSE Paulette, VIDAL Pierre, VOUVÉ Jean, BRUNET Jacques 1994 - La Grotte de Font-de-Gaume : art pariétal, protection, conservation et intervention Périgueux : Fanlac - 1 vol. (48 p.).

GENESTE Jean-Michel, BURAUD Patrice, CHADELLE Jean-Pierre, VOUVÉ Jean, Brunet Jacques et VIDAL Pierre 1995 - Lascaux, état des lieux, mai 1995 - Bordeaux : Direction Régionale des Affaires Culturelles Aquitaine. 1 vol. (100 p.).

GUILLAMET E., BAFFIER D., GIRARD M., BRUNET J. 1997 - Un nouveau mammouth à Arcy-sur-Cure Archéologia, 338, p. 8-9.

GIRARD Michel, BAFFIER Dominique, BRUNET Jacques, GUILLAMET Eudald 2002 - L'Intervention directe sur les parois : un apport à la connaissance des tracés préhistoriques, le cas de la Grande Grotte d'Arcy-sur-Cure (Yonne) - In : L'art avant l'histoire, la conservation de l'art préhistorique / 10èmes journées d'études de la section française de l'institut international de conservation. Paris, 23-24 mai 2002 - Champs-sur-Marne : SFIIC. - p. 197-207.

LORBLANCHET Michel, DEMAILLY Sylvie et BRUNET Jacques 2000 - Les Mystères de la grotte Mazet à Cougnac (Lot). [Suivi d'une annexe] Analyse des concrétions pariétales des grottes de la colline de Cougnac (Lot). Bulletin de la Société des études littéraires, scientifiques et artistiques du Lot, 121, fasc. 4, p. 275-320.

MALAURENT Philippe, LASTENNET Laurent et BRUNET Jacques 2006 - Une grotte sous influence. L'environnement hydrologique et climatique. Monumental, 2006), p. 88-93.

MALAURENT Philippe, BRUNET Jacques, LASTENNET Roland, LOPEZ Benjamin 2008 - Suivi de l'état hydrique d'une paroi de la salle des Taureaux de Lascaux. International Newsletter on Rock Art, vol. 51, p. 16-20.

MALAURENT Philippe, BRUNET Jacques, LASTENNET Roland, LOPEZ Benjamin 2008 - Suivi de l'état hydrique d'une paroi de la salle des Taureaux de Lascaux. Lettre internationale d'informations sur l'art rupestre, 51, p. 16-20.

MALAURENT Philippe, BRUNET Jacques, LACANETTE Delphine, CALTAGIRONE Jean-Paul, GENESTE JeanMichel, SIRE Marie-Anne 2007 - Modélisation numérique et conservation des biens culturels : application à la grotte de
Lascaux - International Newsletter on Rock Art, vol. 48, p. 14-19.

MALAURENT Philippe, LACANETTE Delphine, BRUNET Jacques, RISS Joëlle 2011 - Climatologie du milieu souterrain à Lascaux = Climatology of the subterranean environment at Lascaux : from a global study to the microclimatology of the cave walls : d'une étude globale à la microclimatologie - In : Lascaux et la conservation en milieu souterrain : actes du symposium international, Paris, 26 et 27 février 2009. Paris : Éditions de la Maison des sciences de l'homme, p. 121-142.

RIGAUD Jean-Philippe, VIALOU Denis, AUJOULAT Norbert, LEROI-GOURHAN Arlette, DELLUC Brigitte, DELLUC Gilles, LORBLANCHET Michel, ROUSSOT Alain, ANDRIEUX Claude, BRUNET Jacques, VIDAL P., VOUVÉ Jean, ALLAIN Jacques et RIGAUD André 1990 - Lascaux : Le premier chef-d'œuvre de l'humanité. - Dossiers d'Archéologie, 152, 84 p.

THORN Andrew et BRUNET Jacques 1995 - Preservation of rock art. Second international congress of the Australian rock art research association, Cairns, 1992 - Melbourne : Australian Rock Art Research Association Inc. -1 vol. (230 p.).

VIDAL Pierre, BRUNET Jacques, DAUGLAS Isabelle et al. 1990 - La Conservation de l'art des cavernes et des abris Champs-sur-Marne : Section française de l'Institut international de conservation. - 1 vol. (31 p.-8 p. de pl.).

VIDAL Pierre, BRUNET Jacques et ROUSSOTLARROQUE Julia 1987 - En Périgord Noir. La Roque Saint-Christophe. Première falaise troglodytique d'Occident - Peyzac-le-Moustier : Editions La Roque SaintChristophe. - 1 vol. (30 p.).

VOUVÉ Jean, BRUNET Jacques, MALAURENT Philippe 2000 - Essai sur les perspectives d'analyse insolite du champ graphique pariétal à travers l'effet « miroir » de l'eau identifiée dans la grotte Chauvet - Préhistoire ariégeoise, 55, p. 5-10.

VOUVÉ Jean, BRUNET Jacques et VIDAL Pierre 1985 Contribution de la photogrammétrie et de la thermographie à courte distance à la documentaton systématique des oeuvres pariétales préhistoriques en vue de leur sauvegarde. Application à la grotte de Lascaux - Bulletin de la Société Française de Photogrammétrie et de Télédétection, $\mathrm{n}^{\circ} 98$ (1985-2), p. 23-34.

VOUVÉ Jean, BRUNET Jacques et VIDAL Pierre 1989 Lascaux 1988 : une colline, une grotte ornée, un fac-similé et ... 400000 visiteurs par an (Dordogne, France) Karstologia, 13, p. 33-40.

VOUVÉ Jean, MALAURENT Philippe, BRUNET Jacques 1994 - Interactivité paléothermique, thermique et modalités de bonne conservation naturelle de l'art rupestre Préhistoire ariégeoise, 49, p. 225-230. 
VOUVÉ Jean, MALAURENT Philippe et BRUNET Jacques 1995 - A propos de la stabilité thermique actuelle et passée (quaternaire récent) du milieu karstique- Aide à la sauvegarde de l'héritage culturel préhistorique souterrain. In : Preservation of rock art. Second international congress of the Australian rock art research association, Cairns, 1992 / Andrew Thorn et Jacques Brunet. - Melbourne : Australian Rock Art Research Association Inc. - p. 32-38.

VOUVÉ Jean, MALAURENT Philippe et BRUNET Jacques 1995 - Apport de la dynamique heterothermique à la conservation du patrimoine rupestre en milieu carbonate : le cas de Lascaux. In: Preservation of rock art. Second international congress of the Australian rock art research association, Cairns, 1992 / Andrew Thorn et Jacques Brunet. - Melbourne : Australian Rock Art Research Association Inc. - p. 40-48.

VOUVÉ Jean, MALAURENT Philippe, BRUNET Jacques 1996 - Caractérisation physique et environnementale d'un sanctuaire préhistorique sous-marin, semi-noyé et orné préalablement à l'élaboration d'une démarche conservatoire : cas de la grotte Cosquer, France - Comptes rendus de l'Académie des sciences. Série 2a, 322, fasc. 11, p. 935-942.
VOUVÉ Jean, BRUNET Jacques, VIDAL Pierre, MARSAL Jacques 1982 - Lascaux en Périgord noir - Périgueux : Fanlac. - 1 vol. (87 p.).

VOUVÉ Jean, BRUNET Jacques, VIDAL Pierre et MARSAL Jacques 1983 - Les oeuvres rupestres de Lascaux (Montignac, France) - Londres : International Institute for Conservation of Historic and Artistic Works (Studies in Conservation ; 28). - p. 107-116.

VOUVÉ Jean, BRUNET Jacques, VIDAL Pierre, MARSAL Jacques 1984 - La Grotte de Lascaux sauvegardée Orthez : Lo Trebuc. - 1 vol. (32 p.).

VOUVÉ Jean, VOUVÉ Florence, BRUNET Jacques, MALAURENT Philippe 2000 - Apport de l'analyse colorimétrique à propos de la caractérisation in situ des peintures préhistoriques dans une démarche conservatoire : cas de la grotte Chauvet, Ardèche, France - Comptes rendus de l'Académie des sciences. Série 2a, Sciences de la terre et des planètes, 331 fasc. 9. p. 627-632. 
